\title{
Inteligencia emocional y personalidad: predicción de los niveles de ansiedad en alumnos del Grado en Educación Infantil y Educación Primaria
}

\section{María Isabel Gómez Núñez ${ }^{1}$, María Angeles Cano Muñoz ${ }^{1}$}

${ }^{1}$ Universidad Católica San Antonio de Murcia

\section{España}

Corresponding author: María Ángeles Cano Muñoz, Universidad Católica de Murcia. E-mail: macano@ucam.edu

(C) Universidad de Almería and Ilustre Colegio Oficial de la Psicología de Andalucía Oriental (Spain) 


\section{Resumen}

Introducción. La ansiedad es una emoción que se caracteriza por un conjunto de síntomas cognitivos, psicofisiológicos y conductuales, emitidos por un sujeto ante algunas situaciones que se perciben como amenazantes o peligrosas. El objetivo del presente trabajo fue conocer la relación existente entre ansiedad, inteligencia emocional y personalidad, así como la capacidad predictora de la inteligencia emocional y la personalidad sobre la ansiedad.

Método. Para llevar a cabo este estudio se reclutó una muestra de 140 participantes con edades comprendidas entre los 19 y 28 años de edad $(M=22.57, D T=2.16)$, pertenecientes al Grado en Educación Infantil y Educación Primaria de dos universidades españolas. La muestra de estudio completó el Emotional Quotient Inventory, Trait Meta-Mood-24 Scale, Inventario de Personalidad NEO PI-R e Inventario de Situaciones y Respuestas de Ansiedad.

Resultados: Los análisis de correlación revelaron la existencia de relaciones estadísticamente significativas entre los constructos evaluados (inteligencia emocional, personalidad y ansiedad). Por otro lado, el análisis de regresión múltiple de tipo jerárquico mostró que los factores claridad emocional e inteligencia intrapersonal de la dimensión inteligencia emocional, y los factores neuroticismo y extraversión de la dimensión personalidad, predecían significativamente los niveles de ansiedad.

Discusión y conclusiones. La evaluación de los factores vinculados a la ansiedad favorece la planificación de programas que mejoren su tratamiento y prevención.

Palabras Clave: ansiedad, inteligencia emocional, personalidad, universitarios. 


\begin{abstract}
Introduction. Anxiety is an emotion which is characterized by a set of cognitive, psychophysical and behavioral symptoms, showed by a subject before some situations which are perceived as threatening or dangerous. The aim of the present work was to know the relationship between anxiety, emotional intelligence and personality, as well as the predictive capacity of emotional intelligence and personality on anxiety.
\end{abstract}

Method. To carry out this study, a sample of 140 participants aged between 19 and 28 years old $(M=22.57, S D=2.16)$, belonging to Childhood and Primary Education Degrees of two Spanish universities was recruited. The study sample completed Emotional Quotient Inventory, Trait Meta-Mood Scale-24, NEO PI-R Personality Inventory and Anxiety Situations and Answers Inventory.

Results. The correlation analysis revealed the existence of statistically significant relations between the constructs evaluated (emotional intelligence, personality and anxiety). On the other hand, multiple hierarchical regression analysis showed that some of the dimensions of emotional intelligence and personality significantly predicted anxiety levels.

Discussion or Conclusion. The evaluation of the factors linked to anxiety favors the planning of programs which improve its treatment and prevention.

Keywords: anxiety, emotional intelligence, personality, undergraduates. 


\section{Introducción}

Los trastornos vinculados con la ansiedad se han convertido en una de las patologías con mayores índices de prevalencia en la actualidad en España (De Pedro Cuesta, Ruiz, Roca, \& Noguer, 2016; Ministerio de Economía y Competitividad \& Ministerio de Sanidad, Servicios Sociales e Igualdad, 2018; Ruiz-Rodríguez et al., 2017). La ansiedad, como respuesta emocional compleja, se define como un conjunto de reacciones cognitivas, psicofisiológicas y motoras que se manifiesta ante situaciones que el individuo evalúa como amenazantes y/o ambiguas, aunque objetivamente no lo sean (Cano-Vindel, 2003). En esta definición, se tienen en cuenta dos teorías principales: por un lado, la Teoría Trimensional de Lang $(1968,1985)$, que establece que estas tres reacciones pueden manifestarse de manera parcialmente independiente, aunque también son altamente interactivas entre sí. Esta teoría se ha convertido en uno de los paradigmas más empleados a la hora de estudiar, comprender y tratar emociones como el miedo o la ansiedad (Lawyer \& Smitherman, 2004). Sus postulados han seguido confirmándose y han sido apoyados por investigaciones más recientes (Barlow, 2002; Barlow, Allen, \& Choate, 2004; Martínez-Monteagudo, Inglés, Cano-Vindel, \& GarcíaFernández, 2012). Por otro lado, la variación en los niveles de las reacciones que componen la ansiedad también depende de la situación ante la que se enfrente el individuo tal y como establece la Teoría Interaccionista de Endler (1975), siendo las más importantes aquellas vinculadas con la evaluación, las relaciones interpersonales, los estímulos fóbicos y las situaciones de la vida cotidiana (Miguel-Tobal \& Cano-Vindel, 1997).

Uno de los factores que influye en la manifestación de ansiedad es la personalidad de los individuos. En este sentido, Shi, Liu, Wang y Wang (2015), en su trabajo con estudiantes chinos de Medicina, demostraron que esta se vinculaba positivamente con el neuroticismo y negativamente con la extraversión, la amabilidad, la apertura a la experiencia y la responsabilidad. En el caso de España, el estudio de Caballo, Salazar, Irurtia, Arias y Guillén (2010), con una muestra de estudiantes universitarios españoles, mostraba la relación estadísticamente significativa y positiva de los niveles de ansiedad con el neuroticismo y negativa con el factor extraversión. Del mismo modo, Vazsonyi, Ksinan, Mikuška y Jiskrova (2015), en un estudio en el que empleaban muestras de estudiantes procedentes de distintos países hallaron que, en el caso de España, los niveles de ansiedad solo se vinculaban 
negativamente con el neuroticismo, pero no con los cuatro factores de la personalidad restantes.

Otro de los factores relacionados con los niveles de ansiedad es la inteligencia emocional, la cual ha sido definida desde dos paradigmas distintos: el modelo cognitivo y el modelo mixto. Siguiendo el modelo cognitivo propuesto por Mayer y Salovey (1997), la inteligencia emocional es entendida como un conjunto de habilidades para identificar, comprender, usar y regular las emociones, la cual puede ser autopercibida (Salovey, Mayer, Goldman, Turvey, \& Palfai, 1995) o medida a través de pruebas específicas de habilidad (Mayer, Salovey, \& Caruso, 2002). La ansiedad, según este modelo, se vincula con mayores índices de atención emocional (Cazalla-Luna \& Molero, 2014; Lizeretti, Extremera, \& Rodríguez, 2012) y con una claridad o comprensión y regulación emocional más reducidas (Bourdier, Ringuenet, Duclos, Ringuenet, \& Berthoz, 2016).

Por otro lado, desde el modelo mixto, la inteligencia emocional es definida como un conjunto de habilidades o capacidades sociales, emocionales y personales que influyen en la efectividad a la hora de enfrentarse a las demandas y presiones ambientales (Bar-on, 1997). Dentro de este modelo, se incluyen habilidades como las intrapersonales e interpersonales, adaptabilidad, manejo del estrés y estado anímico. Lu, Li, Hsu y Williams (2010), a través de una muestra de atletas adultos taiwaneses, obtuvieron que la inteligencia intrapersonal e interpersonal y el manejo del estrés actuaban como variables predictoras de la intensidad de la respuesta de ansiedad. Igualmente, Killgore, Sonis, Rosso y Rauch (2016), con una muestra de adultos americanos, hallaron que la ansiedad podría incrementarse ante la presencia de una mayor sensibilidad ante la manifestación de esta emoción, así como con una menor inteligencia emocional general y concretada en las siguientes escalas: inteligencia intrapersonal, manejo del estrés, ánimo general y adaptabilidad.

La inteligencia emocional y la personalidad son constructos relacionados que, en ocasiones, se han solapado o confundido por las correlaciones que existen entre ambos, así como por la presencia de factores similares en el modelo mixto de la inteligencia emocional y los elementos que componen los cinco grandes factores de la personalidad. Di Fabio y Saklofske (2014) mostraron, en una muestra de jóvenes italianos, la existencia de correlaciones positivas y estadísticamente significativas entre los cinco grandes factores de la personalidad y la inteligencia emocional evaluada desde el modelo mixto. Estos resultados 
fueron replicados por Webb et al. (2013) en una muestra de adultos americanos, revelando correlaciones estadísticamente significativas entre los distintos factores de la personalidad y la inteligencia emocional total medida con el Bar-On Emotional Quotient Inventory (EQ-i; BarOn, 2002).

Atendiendo a los resultados hallados según el modelo cognitivo, Salguero, FernándezBerrocal, Balluerka y Aritzeta (2010), empleando una muestra de adolescentes españoles, evidenciaron correlaciones estadísticamente significativas y positivas entre la claridad y la reparación emocional y los cinco factores de personalidad. El factor atención emocional correlacionaba positivamente con el neuroticismo y negativamente con el resto de factores de la personalidad. Por otro lado, Peláez-Fernández, Extremera y Fernández-Berrocal (2014), en un estudio realizado con jóvenes y adultos españoles, mostraron que la amabilidad, la extraversión, el neuroticismo y la apertura a la experiencia interactuaban con la atención, la claridad y la reparación emocional en la predicción de la agresión (física, verbal, ira, hostilidad y agresión total).

La determinación y comprensión de los factores que podrían influir en el aumento de los niveles de ansiedad resulta esencial para prevenir trastornos más graves que impidan el desempeño diario de una persona (Gustems-Carnicer, Calderón, \& Forn-Santacana, 2017). Los estudios realizados con universitarios se han centrado, en mayor medida, en la evaluación de la ansiedad ante los exámenes (Álvarez, Aguilar, \& Lorenzo, 2012) o en la valoración de los diferentes perfiles socioemocionales que presentan estudiantes de distintas titulaciones académicas (Castejón, Cantero, \& Pérez, 2008). En este sentido, la falta de investigaciones en población universitaria española en la que se evalúen las relaciones entre la inteligencia emocional desde los dos modelos propuestos, la personalidad y los niveles de ansiedad teniendo en cuenta la complejidad de esta respuesta emocional, justifican la importancia de este estudio. De hecho, estudios realizados en otros contextos como el australiano demuestran que la inteligencia emocional predice resultados, en relación al ajuste personal, que van más allá de las contribuciones realizadas por la personalidad (Bastian, Burns \& Nettelbeck, 2005). La comprensión de estas relaciones resulta básica para prevenir trastornos emocionales vinculados con la ansiedad. 


\section{Objetivos e hipótesis}

A partir de las consideraciones teóricas anteriormente expuestas, el objetivo principal de este estudio es analizar la relación entre ansiedad, personalidad e inteligencia emocional medida desde el modelo cognitivo y el modelo mixto, así como la capacidad predictora de la inteligencia emocional y de la personalidad sobre la ansiedad general en alumnos del Grado en Educación Infantil y Educación Primaria. Específicamente esperamos que: a) el factor neuroticismo correlacionará positiva y significativamente con los tipos de ansiedad evaluados; b) los factores amabilidad, responsabilidad, apertura a la experiencia y extraversión correlacionarán negativa y significativamente con los niveles de ansiedad; c) la atención emocional correlacionará positiva y significativamente con los niveles de ansiedad; d) la claridad y reparación emocional correlacionarán negativa y significativamente con la ansiedad; e) la inteligencia intrapersonal, manejo del estrés, ánimo general y adaptabilidad correlacionan negativa y significativamente con la ansiedad; f) la personalidad y la inteligencia emocional explican de manera independiente los niveles de ansiedad general

\section{Método}

\section{Participantes}

Los alumnos objeto de estudio pertenecen a dos universidades privadas española, seleccionadas por la accesibilidad a las mismas. Posteriormente, a través de un muestreo aleatorio estratificado realizado a la población de estudiantes de los Grados en Educación Infantil y Educación Primaria, seleccionamos a los participantes de nuestra investigación, de manera que las dos universidades y los distintos cursos estuviesen representados proporcionalmente. La muestra final estuvo formada por un total de 140 hombres y mujeres con edades comprendidas entre los 19 y 28 años de edad $(M=22.57, D T=2.16)$. La Tabla 1 muestra la distribución de estudiantes por universidades, el porcentaje de hombres y mujeres, el rango de edad, así como la media y la desviación típica en función de esta última variable.

Tabla 1. Estadísticos descriptivos de la muestra por universidades

\begin{tabular}{ccccccc}
\hline Universidad & N & Hombres & Mujeres & Rango de edad & $M($ edad) & DT (edad) \\
\hline 1 & 70 & $44 \%$ & $54 \%$ & $19-25$ & 22.12 & 2.05 \\
2 & 70 & $42 \%$ & $58 \%$ & $19-28$ & 23.27 & 2.59 \\
\hline
\end{tabular}




\section{Instrumentos}

Inventario de Situaciones y Respuestas de Ansiedad (ISRA; Miguel-Tobal \& CanoVindel, 1997). Autoinforme que consta de 24 respuestas de ansiedad y 22 situaciones ansiógenas. Las respuestas de ansiedad se agrupan en tres factores: Ansiedad Cognitiva (reacciones mentales, vinculadas con el pensamiento), Ansiedad Motora (reacciones conductuales observables) y Ansiedad Fisiológica (reacciones provocadas por el Sistema Nervioso Autónomo y Somático, algunas de carácter involuntario). Las situaciones ansiógenas se agrupan en cuatro factores: Evaluación (ansiedad manifestada ante situaciones en las que el sujeto puede sentirse evaluado o criticado), Relaciones Interpersonales (ansiedad manifestada ante situaciones sexuales y de interacción social), Estímulos Fóbicos (ansiedad manifestada ante determinados estímulos) y Situaciones de la Vida Cotidiana (ansiedad manifestada ante hechos diarios o comunes). Cada situación se valora en función de las distintas respuestas propuestas, haciendo un total de 224 ítems. Los autores informan de una adecuada validez de constructo y una buena capacidad de discriminación entre grupos y patologías. Esta prueba permite adecuar el tratamiento de ansiedad al tipo de manifestación y de situación en la que se presenta. La consistencia interna (Alpha de Cronbach) para este estudio osciló entre .83-.89.

Trait Meta-Mood Scale-24 (TMMS-24; Fernández-Berrocal, Extremera, \& Ramos, 2004). Inventario compuesto por 24 ítems agrupados en tres factores: Atención (grado en el que se presta atención a las propias emociones), Claridad (habilidad para evaluar, discriminar y comprender los propios estados emocionales) y Reparación (habilidad para regular nuestro estado emocional). Este instrumento permite la evaluación de la inteligencia emocional percibida por el propio sujeto evaluado. Presenta buenas propiedades psicométricas, oscilando la consistencia interna (Alpha de Cronbach) para este estudio entre .81-.85.

Emotional Quotient Inventory: Short Form (EQ-i:S; Bar-On, 2002). Autoinforme compuesto por 51 ítems derivado del original con 133 ítems elaborado por Bar-On (1997). Este instrumento contiene las siguientes escalas: Capacidad Intrapersonal (asertividad, autoconocimiento, autoevaluación, independencia y capacidad de actualizarnos), Habilidades Interpersonales (empatía, habilidades sociales y responsabilidad social), Adaptabilidad (resolución de problemas, evaluación real y flexibilidad), Manejo del Estrés (tolerancia al estrés y control de los impulsos) y Humor General (felicidad y optimismo). Además, incluye una puntuación total de inteligencia emocional y una escala de deseabilidad social. Altas 
puntuaciones en la escala reflejan altos niveles de competencia social y emocional. La consistencia interna (Alpha de Cronbach) para este estudio osciló entre .77-.86.

Inventario de personalidad NEO Revisado (NEO PI-R; Costa \& McCrae, 1992). Instrumento formado por 240 ítems agrupados en cinco grandes factores de personalidad: Neuroticismo (grado de inestabilidad emocional), Extraversión (grado de sociabilidad, atrevimiento, tendencia a estar con los demás, etc.), Apertura a la Experiencia (apertura al cambio, imaginación, curiosidad, etc.), Responsabilidad (grado de planificación, autocontrol, organización) y Amabilidad (tendencias interpersonales de carácter positivo). La consistencia interna (Alpha de Cronbach) de la prueba para este estudio osciló entre .76-.83.

\section{Procedimiento}

Una vez realizado el proceso de selección de la muestra de participantes y tras la obtención del consentimiento informado de los mismos, se procedió a la administración de las pruebas. Para ello, se elaboró un cuadernillo con los cuatro instrumentos de medida, con el fin de facilitar la tarea a los sujetos y también para evitar pérdidas o la devolución de los cuestionarios incompletos.

La aplicación de las pruebas se realizó de manera colectiva en las aulas de clase. Las pruebas se administraron en dos sesiones de una hora durante dos días consecutivos, con el fin de evitar el agotamiento de los participantes y su atención a la hora de completar los instrumentos.

Una vez completadas las pruebas, a cada uno de los grupos se les agradeció de manera conjunta su colaboración y participación en el proyecto, asegurando la entrega de resultados a aquellas personas que así lo habían pedido. Así mismo, las investigadoras se ofrecieron a resolver todas aquellas dudas que pudiesen existir ofreciendo, además, dos sesiones de intervención para la mejora de la inteligencia emocional y el afrontamiento de los problemas de ansiedad.

\section{Análisis de datos}

La consistencia interna de las puntuaciones obtenidas con los distintos instrumentos fue calculada mediante coeficientes Alpha de Cronbach. George y Mallery (2003) sugieren las siguientes recomendaciones para evaluar estos coeficientes: menor que 0.5 se consideraría 
inaceptable, entre 0.5 y 0.6 pobre, entre 0.6 y 0.7 cuestionable, entre 0.7 y 0.8 aceptable, entre 0.8 y 0.9 bueno y valores iguales o superiores a 0.9 se consideraría excelente.

Para analizar las correlaciones entre los distintos factores del IAEP, se calcularon los coeficientes de correlación producto-momento de Pearson. Para coeficientes de correlación, Cohen (1988) sugirió que valores iguales o mayores que .10 e inferiores a .30 indican una relación de pequeña magnitud, valores mayores que .30 indican una magnitud moderada, mientras que valores superiores a .50 señalarían correlaciones de magnitud elevada/alta.

Por último, para examinar la influencia de la personalidad y la inteligencia emocional sobre los niveles de ansiedad escolar, se utilizó el análisis de regresión múltiple a través del método jerárquico, que permite determinar la contribución específica de posibles variables predictoras sobre una variable criterio, controlando el efecto de las variables introducidas previamente en la ecuación. Así, las variables que se introducen en primer lugar actuarán como covariantes de las que se incluyen posteriormente de forma que, solo en el último paso, podríamos establecer la contribución única a la explicación de la variable criterio. La decisión de introducir unas variables antes que otras, depende de la base teórica asumida. En este estudio el diagnóstico para la independencia de los errores fue aceptable ( $D W=2)$ y la colinealidad adecuada (FIV = 1.9-5.6), lo que otorga validez al presente análisis. Para los análisis de datos propuestos se utilizó paquete estadístico SPSS 22.

\section{Resultados}

\section{Análisis de correlación}

En la Tabla 2 se muestran las distintas correlaciones entre las variables estudiadas. Los análisis de las relaciones entre los factores de ansiedad (ISRA), los factores de personalidad (NEO PI-R) y las escalas de inteligencia emocional incluidas en el TMMS y EQ-i:S, revelaron la existencia de correlaciones estadísticamente significativas de rango moderadoalto entre algunas de las escalas de dichos instrumentos. Las distintas dimensiones de la ansiedad (situacional y respuestas) correlacionan positiva y significativamente con el factor Neuroticismo, con una magnitud baja-moderada de dichas relaciones $(r=.38-.70)$. El factor Extraversión correlacionaba negativa y significativamente con todos los tipos de ansiedad, oscilando entre una magnitud baja y moderada $(r=.39-.73)$. Del mismo modo, se destaca el factor Intrapersonal que correlacionaba negativa y significativamente con todas las 
dimensiones de la ansiedad. La magnitud de estas correlaciones osciló entre un nivel bajo ( $r=$ $.43)$ y moderado $(r=.62)$.

Tabla 2. Correlación entre los distintos factores de ansiedad (IAEP), personalidad (NEO PI $R)$ e inteligencia emocional (TMMS y EQ-i:S)

\begin{tabular}{|c|c|c|c|c|c|c|c|c|c|c|c|c|c|c|c|c|c|c|c|c|c|c|}
\hline & V1 & $\mathrm{V} 2$ & V3 & V4 & V5 & V6 & V7 & V8 & V9 & V10 & V11 & V12 & V13 & V14 & V15 & V16 & V17 & V18 & V19 & $\mathrm{V} 20$ & $\mathrm{~V} 21$ & $\mathrm{~V} 22$ \\
\hline 1. A.Cognitiva & 1.00 & & & & & & & & & & & & & & & & & & & & & \\
\hline 2. A.Motora & $.74 * *$ & 1.00 & & & & & & & & & & & & & & & & & & & & \\
\hline 3. A.Fisiológica & $.83^{* *}$ & $.64 * *$ & 1.00 & & & & & & & & & & & & & & & & & & & \\
\hline 4. A.Evaluación & $.90^{* *}$ & $.76 * *$ & $.86^{* *}$ & 1.00 & & & & & & & & & & & & & & & & & & \\
\hline 5. A.Interpersonal & $.78^{* * *}$ & $.68 * *$ & $.82 * *$ & $.73 * *$ & 1.00 & & & & & & & & & & & & & & & & & \\
\hline 6. A.Fóbica & $.75^{* * *}$ & $.69^{* *}$ & $.81^{* *}$ & $.71 * *$ & $.63^{* *}$ & 1.00 & & & & & & & & & & & & & & & & \\
\hline 7. A.Cotidiana & $.73^{* *}$ & $.76 * *$ & $.73^{* *}$ & $.72 * *$ & $.64 * *$ & $.53 * *$ & 1.00 & & & & & & & & & & & & & & & \\
\hline 8. A.Total & $.95^{* * *}$ & $.86^{* *}$ & $.90 * *$ & $.93^{* *}$ & $.83^{* *}$ & $.82 * *$ & $.81 * *$ & 1.00 & & & & & & & & & & & & & & \\
\hline 9. Neuroticismo & $.64^{* *}$ & $.65^{* *}$ & $.55^{* *}$ & $.70^{* *}$ & $.53^{* *}$ & $.38^{*}$ & $.57^{* *}$ & $.67 * *$ & 1.00 & & & & & & & & & & & & & \\
\hline 10. Extraversión & $-.62 * *$ & $-.61^{* *}$ & $-.56^{* *}$ & $-.59 * *$ & $-.73 * *$ & $-.39 *$ & $-.52 * *$ & $-.66^{* *}$ & $-.63^{* *}$ & 1.00 & & & & & & & & & & & & \\
\hline 11. Apertura & -.23 & -.17 & $-.32 *$ & -.17 & $-.36^{*}$ & -.20 & -.12 & -.26 & $-.37 *$ & $.59 * *$ & 1.00 & & & & & & & & & & & \\
\hline 12. Amabilidad & .10 & -.19 & -.01 & -.05 & -.03 & .03 & -.07 & -.01 & -.24 & .05 & -.06 & 1.00 & & & & & & & & & & \\
\hline 13. Responsabilidad & .12 & -.10 & .21 & .08 & .13 & .03 & .02 & .09 & .07 & -.13 & -.28 & .24 & 1.00 & & & & & & & & & \\
\hline 14. Atención & .03 & -.03 & -.21 & .13 & -.001 & -.01 & .25 & .07 & -.03 & .27 & $.39^{*}$ & .20 & .22 & 1.00 & & & & & & & & \\
\hline 15. Claridad & $-.34^{*}$ & -.18 & $-.33^{*}$ & $-.36^{*}$ & $-.33^{*}$ & -.10 & -.29 & $-.32 *$ & $-.53^{* *}$ & $.53 * *$ & $.37 *$ & .23 & .05 & .15 & 1.00 & & & & & & & \\
\hline 16. Reparación & -.15 & -.18 & -.12 & -.22 & -.13 & -.02 & -.16 & -.16 & $-.54 * *$ & $.50^{* *}$ & $.34^{*}$ & $.40^{* *}$ & .24 & $.38 *$ & $.59 * *$ & 1.00 & & & & & & \\
\hline 17. Intrapersonal & $-.62 * *$ & $-.51 * *$ & $-46^{* *}$ & $-.56 * *$ & $-.45^{* *}$ & $-.43 * *$ & $-.43^{* *}$ & $-.59 * *$ & $-.63^{* *}$ & $.51^{* *}$ & $.46^{* *}$ & -.16 & .01 & .04 & $.52 * *$ & .18 & 1.00 & & & & & \\
\hline 18. Interpersonal & .20 & -.03 & $-.34 *$ & .21 & .05 & $.32 *$ & .17 & .19 & -.02 & .21 & .07 & $.45^{* *}$ & $.33^{*}$ & $.34 *$ & -.01 & $.50 * *$ & -.22 & 1.00 & & & & \\
\hline $\begin{array}{l}\text { 19. Manejo del } \\
\text { estrés }\end{array}$ & $-.31 *$ & $-.33^{*}$ & -.25 & -.23 & -.20 & -.30 & -.22 & $-.33^{*}$ & $-.46^{* *}$ & .05 & .09 & $.34 *$ & .04 & -.05 & .11 & .12 & $.35^{*}$ & -.003 & 1.00 & & & \\
\hline 20. Adaptabilidad & -.14 & -.03 & .23 & .17 & .11 & .12 & .03 & .13 & .22 & -.06 & $-.33^{*}$ & .17 & $.55^{* *}$ & .02 & .20 & .01 & -.21 & $.34 *$ & -.17 & 1.00 & & \\
\hline 21. Humor & $-.40 * *$ & -.30 & $-48^{* *}$ & $-.47 * *$ & -.29 & -.26 & -.28 & $-.43^{* *}$ & $-.60 * *$ & $.52 * *$ & $.49 * *$ & .19 & -.21 & -.02 & $.56^{* *}$ & $.58 * *$ & $.41 * *$ & .03 & .20 & .19 & 1.00 & \\
\hline 22. EQi_TOTAL & $-.45^{* *}$ & $-.52 * *$ & -.31 & $-.41 * *$ & $-.34^{*}$ & -.27 & $-.33^{*}$ & $-.47 * *$ & $-.66^{* *}$ & $.52 * *$ & $.36^{*}$ & $.37 *$ & .24 & .12 & $.43^{* *}$ & $.55^{* *}$ & $.61^{* *}$ & $.38^{*}$ & $.61^{* *}$ & .20 & $.62^{* *}$ & 1.00 \\
\hline$M(\mathrm{~N}=140)$ & 85.00 & 54.88 & 51.10 & 84.38 & 22.83 & 47.33 & 18.55 & 190.98 & 36.25 & 40.53 & 39.25 & 41.63 & 40.80 & 24.48 & 24.35 & 26.40 & 33.88 & 39.35 & 27.80 & 25.33 & 34.88 & 32.25 \\
\hline$D T$ & 38.59 & 27.49 & 29.16 & 34.73 & 14.81 & 30.66 & 13.74 & 87.10 & 6.72 & 6.66 & 8.40 & 6.49 & 5.91 & 5.67 & 4.32 & 6.07 & 5.12 & 4.12 & 4.87 & 3.79 & 4.60 & 2.27 \\
\hline
\end{tabular}

$* p=<.05 ; * * p=<.01 ; \mathrm{A}=$ Ansiedad; $\mathrm{V}=$ Variable; Neuroticismo, Extraversión, Apertura, Amabilidad, Responsabilidad (NEO-personalidad); Atención, Claridad, Reparación (TMMS); Inteligencia Intrapersonal, Inteligencia Interpersonal, Manejo del Estrés, Adaptabilidad, Humor, EQi Total (EQi)

\section{Análisis de regresión jerárquica}

En la Tabla 3 aparecen los resultados referentes a los análisis de regresión múltiple de tipo jerárquico, tomando como criterio la puntuación total de ansiedad.

En primer lugar, incluimos en la ecuación el bloque de las variables relativas a los cinco factores de personalidad (neuroticismo, extraversión, apertura a la experiencia, amabilidad y responsabilidad). La contribución de este bloque a la predicción de la ansiedad fue estadísticamente significativa $(\mathrm{R}=.77, \mathrm{~F}=9.88, p<.001)$. Con la introducción del segundo bloque, en el que se incluyeron las variables relativas a la inteligencia emocional tanto desde el modelo cognitivo (TMMS) como desde el modelo mixto (EQ-i:S), volvimos a obtener datos que manifestaban una contribución significativa de las variables predictoras a la explicación de la variable criterio $(\mathrm{R}=.88, \mathrm{~F}=7.15, p<.001)$. El porcentaje de explicación de la 
varianza de la variable criterio en el primer bloque fue estadísticamente significativo $\left(\mathrm{R}^{2}=.59\right.$, $\mathrm{p}<.001$ ), aumentando también de manera significativa con la incorporación del segundo bloque relativo a la inteligencia emocional $\left(\mathrm{R}=.78, \Delta \mathrm{R}^{2}=.18, p=.022\right)$.

Tabla 3. Análisis de regresión jerárquica para la ansiedad general

\begin{tabular}{|c|c|c|c|c|c|c|c|}
\hline Variable & $\boldsymbol{R}$ & $R^{2}$ & $B$ & $S E B$ & $\beta$ & $F$ & $p$ \\
\hline Paso 1 & .77 & .59 & & & & & \\
\hline Neuroticismo & & & 6.17 & 1.90 & $.47 * *$ & 9.88 & $<.001$ \\
\hline Extraversión & & & -6.50 & 2.13 & $-.49 * *$ & & \\
\hline Apertura a la experiencia & & & 2.29 & 1.46 & .22 & & \\
\hline Amabilidad & & & 1.77 & 1.58 & .13 & & \\
\hline Responsabilidad & & & .33 & 1.73 & .02 & & \\
\hline Paso 2 & .88 & .77 & & & & 7.15 & $<.001$ \\
\hline Neuroticismo & & & 2.66 & 2.58 & $.38 * *$ & & \\
\hline Extraversión & & & -11.18 & 2.23 & $-.43 * *$ & & \\
\hline Apertura a la experiencia & & & 2.18 & 1.57 & .21 & & \\
\hline Amabilidad & & & -1.90 & 1.94 & -.14 & & \\
\hline Responsabilidad & & & -3.20 & 2.19 & -.21 & & \\
\hline Atención Emocional & & & 1.11 & 2.00 & .07 & & \\
\hline Claridad Emocional & & & 6.42 & 3.38 & $-.31 *$ & & \\
\hline Regulación Emocional & & & 2.23 & 3.12 & .15 & & \\
\hline Inteligencia intrapersonal & & & -2.44 & 3.21 & $-.34 *$ & & \\
\hline Inteligencia interpersonal & & & 7.36 & 3.16 & .14 & & \\
\hline Manejo del Estrés & & & -1.74 & 2.27 & -.09 & & \\
\hline Adaptabilidad & & & 2.56 & 3.02 & .11 & & \\
\hline Humor general & & & -3.04 & 3.05 & -.16 & & \\
\hline
\end{tabular}

${ }^{*} p=<.05 ; * * p=<.001 ; \Delta R^{2}=.18, p=.022$

No obstante, debemos tener en cuenta que, tras la introducción del primer bloque, solo el factor neuroticismo $(\beta=.47, p=.003)$ y el factor extraversión $(\beta=-.49, p=.004)$ realizaban una aportación significativa a la explicación de la ansiedad. Seguidamente, cuando se incluía el bloque referente a la inteligencia emocional, la claridad emocional (TMMS) ( $\beta=-.34, p=.031)$, la inteligencia intrapersonal (EQ-i:S) $(\beta=.34, p=.028)$ y los factores de personalidad neuroticismo y extraversión $(\beta=.38, p<.001 ; \beta=-.43, p<.001)$ continuaban explicando de manera significativa la ansiedad. 


\section{Discusión y Conclusiones}

El objetivo principal de este estudio fue analizar la relación entre ansiedad, personalidad e inteligencia emocional medida desde el modelo cognitivo y el modelo mixto, así como la capacidad predictora de la inteligencia emocional y de la personalidad sobre la ansiedad general en alumnos del Grado en Educación Infantil y Educación Primaria.

La primera hipótesis, por la que se esperaba que el neuroticismo correlacionara positiva y significativamente con las distintas formas de ansiedad evaluadas, fue confirmada. Estos resultados son similares a los obtenidos en investigaciones previas, en las que el neuroticismo, también denominado inestabilidad emocional, es una variable que se asocia negativamente con la salud mental $\mathrm{y}$, por tanto, positivamente con trastornos como la ansiedad o la depresión (Caballo et al., 2010; Shi et al., 2015; Vazsonyi et al., 2015). La magnitud de las correlaciones (baja-moderada) confirma que hablamos de constructos relacionados, pero distintos. De este modo el incremento de los niveles de neuroticismo aumentaría la probabilidad de manifestar ansiedad en sus distintas dimensiones.

La segunda hipótesis, por la que cabría esperar que los factores extraversión, amabilidad, responsabilidad $\mathrm{y}$ apertura a la experiencia correlacionaran negativa $\mathrm{y}$ significativamente con los niveles de ansiedad, se confirmó parcialmente. Los resultados muestran la existencia de una correlación negativa y estadísticamente significativa entre las distintas formas de ansiedad y el factor extraversión, así como entre el factor apertura a la experiencia y la ansiedad fisiológica y ansiedad ante las relaciones interpersonales, con la ausencia de correlaciones estadísticamente significativas entre el resto de factores de ansiedad y personalidad. Estos resultados, sin embargo, resultan coherentes con lo hallado en investigaciones previas (Caballo et al., 2010; Shi et al., 2015), lo que indica que el nivel de extraversión y de apertura a la experiencia podrían mejorar la salud mental de los individuos al asociarse con una reducción de los niveles de ansiedad. La magnitud baja-moderada de estas correlaciones indicaría que el aumento o disminución de los niveles de ansiedad no depende únicamente de los factores mencionados, aunque resulten importantes en su variación. Igualmente, la ausencia de relaciones con el resto de factores de personalidad, podría deberse a las características de la muestra y al hecho de que factores como la 
amabilidad o la responsabilidad no sean tan determinantes en la aparición de la ansiedad, tal y como muestran investigaciones previas en este ámbito (Vazsonyi et al., 2015).

La tercera hipótesis, según la cual se suponía que la atención emocional correlacionara positiva y significativamente con la ansiedad, no se confirmó. Estos resultados, aunque contradicen lo hallado en otras investigaciones (Cazalla-Luna \& Molero, 2014; Lizeretti et al., 2012), también resultan coherentes con otros estudios en los que la ansiedad y la atención emocional no se vinculan significativamente (Bourdier et al., 2016). En este sentido, es posible que solo los individuos que manifiestan niveles altos de ansiedad sean los que presentan índices mayores de atención o hipervigilancia emocional y general (Bourdier et al., 2016; Grills-Taquechel, Fletcher, Vaughn, Denton, \& Taylor; 2013), mientras que niveles bajos o moderados de ansiedad no tendrían por qué asociarse con un incremento de la atención emocional, como es el caso de la muestra de estudio.

La cuarta hipótesis, por la que se esperaba que la claridad y la reparación emocional correlacionaran negativa y significativamente con la ansiedad, se confirmó parcialmente. Es decir, el factor claridad emocional correlacionaba negativa y significativamente con la ansiedad cognitiva, ansiedad fisiológica, ansiedad ante la evaluación, ansiedad ante las relaciones interpersonales y ansiedad total, siendo coherente con otras investigaciones relevantes sobre esta temática (Bourdier et al., 2016; Cazalla-Luna \& Molero, 2014). La baja magnitud de las correlaciones y la ausencia de correlaciones significativas entre los distintos factores de ansiedad y la regulación emocional puede ser debida a que la muestra presenta niveles medios de ansiedad, lo que no interferiría en la regulación que hagan de sus propias emociones (Martínez-Monteagudo, Inglés, Granados, Aparisi, \& García-Fernández, 2019).

La quinta hipótesis, en la que se preveía que la inteligencia intrapersonal, el manejo del estrés, el estado de ánimo general y la adaptabilidad correlacionaran negativa y significativamente con la ansiedad, se confirmó parcialmente. Los resultaron mostraron la existencia de correlaciones negativas y significativas entre el factor inteligencia intrapersonal y todas las dimensiones de ansiedad, entre los factores ansiedad cognitiva, motora y ansiedad total con el manejo del estrés, entre los factores ansiedad cognitiva, fisiológica, ansiedad ante la evaluación y ansiedad total con el estado anímico general y la ausencia de relaciones con la adaptabilidad. La magnitud baja-moderada de las relaciones existentes muestra la importancia de estas relaciones pero, a su vez, la posible presencia de otras variables mediadoras y de la 
diferenciación existente entre la ansiedad y las variables vinculadas a la inteligencia emocional. Las relaciones mencionadas resultan coherentes con investigaciones sobre esta temática, así como la relación positiva con la inteligencia emocional total medida por el modelo mixto (Killgore et al., 2016). La ausencia de relaciones en algunos de los casos estudiados evidencia que no todas las dimensiones de la inteligencia emocional son determinantes para la presencia de ansiedad en adultos ( $\mathrm{Lu}$ et al., 2010).

Finalmente, la sexta hipótesis, por la que se esperaba que la personalidad y la inteligencia emocional explicaran de manera independiente los niveles de ansiedad general manifestados por la muestra de estudiantes, quedó confirmada para algunos de los factores introducidos en el modelo. El análisis de regresión jerárquica mostró que mayores índices de neuroticismo y menores de extraversión, claridad e inteligencia intrapersonal, predicen significativamente mayores niveles de ansiedad general. Estos resultados indican que, aunque la personalidad y la inteligencia emocional son constructos íntimamente relacionados tanto desde el modelo cognitivo (Peláez-Fernández et al., 2014; Salguero et al., 2010) como desde el modelo mixto (Di Fabio \& Saklofske, 2014; Webb et al., 2013), lo cierto es que son independientes (Pytlik-Zilling, Homenover, \& Dienstbier, 2002). Por lo tanto, podemos establecer que la inteligencia emocional predice resultados en relación al ajuste personal que van más allá de las contribuciones realizadas por la personalidad (Bastian et al., 2005).

Tras los resultados obtenidos, podemos afirmar que algunos de los factores que componen los constructos de personalidad e inteligencia emocional desde los dos modelos estudiados (cognitivo y mixto), presentan correlaciones significativas con la ansiedad destacando, además, que la inteligencia emocional puede predecir la ansiedad con independencia de las aportaciones realizadas por la personalidad, corroborando así las hipótesis planteadas y contribuyendo a la fundamentación teórica sobre las definiciones de inteligencia emocional y personalidad.

No obstante, a pesar de los resultados que confirman las hipótesis y aquellos que aportan nuevas ideas al estudio de las relaciones entre ansiedad, personalidad e inteligencia emocional, no debemos olvidar las limitaciones que presenta este estudio, las cuales deberían ser tenidas en cuenta en futuras investigaciones. En primer lugar, aludimos a la selección de las universidades por accesibilidad. Muestreos de tipo probabilístico por conglomerados, con un mayor número de participantes y abarcando distintas titulaciones, ofrecerían resultados 
más concretos que nos permitirían seguir estudiando las relaciones entre las variables establecidas. En segundo lugar, es posible que en los hallazgos influyan variables extrañas de carácter psicoeducativo que deberían ser controladas para incrementar la validez y fiabilidad de este trabajo. En tercer lugar, sería necesario asumir una perspectiva multimétodo con el fin de contrastar los resultados obtenidos a través de diversas perspectivas, instrumentos o técnicas que incrementaran la información hallada. Por último, resultaría conveniente añadir un análisis de ecuaciones estructurales que nos permitiera analizar, de una manera más amplia y pertinente, los distintos modelos explicativos de la ansiedad, pudiendo establecer relaciones de naturaleza más causal. Estos aspectos deberán ser tenidos en cuenta en estudios que amplíen los resultados de esta investigación.

A pesar de las limitaciones y consideraciones posteriores, este estudio revela la importancia de incluir la inteligencia emocional como variable explicativa de la ansiedad y nos permite adquirir un conocimiento más profundo de aquellas variables relacionadas con su mantenimiento, aumento o disminución en población universitaria española, en concreto, alumnos del Grado en Educación Infantil y Educación Primaria. El estudio de dichas variables resulta imprescindible para evitar el malestar psicológico en población adulta (GustemsCarnicer et al., 2017; Ruiz, Suárez-Falcón, Riaño-Hernández, \& Gillanders, 2017). Además, no debemos obviar que la presencia de los trastornos de ansiedad se ha incrementado considerablemente en la población española durante los últimos años (de Pedro Cuesta, Ruiz, Roca, \& Noguer, 2016; Ministerio de Economía y Competitividad \& Ministerio de Sanidad, Servicios Sociales e Igualdad, 2018). Estos hallazgos proporcionan un conocimiento más exhaustivo de las variables que pueden influir y predecir la ansiedad en sus diferentes manifestaciones, lo que ayudaría en el diseño de programas preventivos, trabajando aspectos relacionados tanto con la inteligencia emocional como con la personalidad, según la dimensionalidad de dichos constructos.

\section{Referencias}

Álvarez, J., Aguilar, J. M., \& Lorenzo, J. J. (2012). La Ansiedad ante los Exámenes en Estudiantes Universitarios: Relaciones con variables personales y académicas. Electronic Journal of Research in Educational Psychology, 10(1), 333-354.

Bar-On, R. (1997). Bar-On Emotional Quotient Inventory (EQ-I): Technical manual. Toronto, Canada: MultiHealth Systems Inc. 
Bar-On, R. (2002). Bar-On Emotional Quotient Inventory: Short version: Technical manual. Toronto, Canada: MultiHealth Systems Inc.

Barlow, D. H. (2002). Anxiety and its disorders: The nature and treatment of anxiety and panic. Nueva York: Guilford.

Barlow, D. H., Allen, L. B., \& Choate, M. L. (2004). Towards a unified treatment for emotional disorders. Behavior Therapy, 35, 205-230.

Bastian, V. A., Burns, N. R., \& Nettelbeck, T. (2005). Emotional intelligence predicts life skills, but not as well as personality and cognitive abilities. Personality and Individual Differences, 39, 1135-1145.

Bourdier, L., Ringuenet, D., Duclos, J., Ringuenet, D., \& Berthoz, S. (2016). Propriétés psychométriques de la version française d'une échelle de mesure de l'intelligence émotionnelle perçue: La trait meta-mood scale (TMMS)/Psychometric properties of the french version of a scale measuring perceived emotional intelligence: The trait metamood scale (TMMS). Canadian Journal of Psychiatry, 61(10), 652. doi: $10.1177 / 0706743716639936$

Caballo, V. E., Salazar, I. C., Irurtia, M. J., Arias, B., \& Guillén, J. L. (2010). Relationships between social anxiety and personality traits, styles, and disorders. Behavioral Psychology-Psicologia Conductual, 18(2), 259-276.

Cano-Vindel, A. (2003). Desarrollos actuales en el estudio del control emocional. Ansiedad y Estrés, 9(2-3), 203-229.

Castejón, J. L., Cantero, M. P., \& Pérez, N. (2008). Diferencias en el perfil de competencias socio-emocionales en estudiantes universitarios de diferentes ámbitos científicos. Electronic Journal of Research in Educational Psychology, 6(2), 339-362.

Cazalla-Luna, N., \& Molero, D. (2014). Inteligencia emocional percibida, ansiedad y afectos en estudiantes universitarios $=$ perceived emotional intelligence, anxiety and affect on university students. REOP-Revista Española De Orientación y Psicopedagogía, 25(3), $56-73$.

Cohen, J. (1988). Statistical power analysis for the behavioral sciences. Hillsdale, NJ: Erlbaum.

Costa, P. T., Jr., \& McCrae, R. R. (1992). Revised NEO Personality Inventory (NEO-PI-R) and NEO Five Factor Inventory (NEO-FFI) professional manual. Odessa, FL: Psychological Assessment Resources. 
De Pedro Cuesta, J., Ruiz, J. S., Roca, M., \& Noguer, I. (2016). Salud mental y salud pública en españa: Vigilancia epidemiológica y prevención. Psiquiatría Biológica, 23(2), 6773.

Di Fabio, A., \& Saklofske, D. H. (2014). Comparing ability and self-report trait emotional intelligence, fluid intelligence, and personality traits in career decision. Personality and Individual Differences, 64, 174-178. doi: 10.1016/j.paid.2014.02.024

Endler, N. S. (1975). A person-situation interaction model for anxiety. En C.A. Spielberger y I.G. Sarason, (eds.), Stress and Anxiety (vol. 1). Washington, D.C.: Hemisphere Publishing.

Fernández-Berrocal, P., Extremera, N., \& Ramos, N. (2004). Validity and reliability of the Spanish modified version of the Trait Meta-Mood Scale. Psychological Reports, 94, 771-775.

Gustems-Carnicer, J., Calderón, C., \& Forn-Santacana, M. (2017). Psychometric properties of the life orientation test (LOT-R) and its relationship with psychological well-being and academic progress in college students. Revista Latinoamericana De Psicología, 49(1), 19-27. doi: 10.1016/j.rlp.2016.09.006

Grills-Taquechel, A. E., Fletcher, J. M., Vaughn, S. R., Denton, C. A., \& Taylor, P. (2013). Anxiety and inattention as predictors of achievement in early elementary school children. Anxiety, Stress \& Coping, 26(4), 391-410.

Killgore, W. D., Sonis, L. A., Rosso, I. M., \& Rauch, S. L. (2016). Emotional intelligence partially mediates the association between anxiety sensitivity and anxiety Symptoms1, 2. Psychological Reports, 118(1), 23-40. doi: 10.1177/0033294115625563

Lang, P. J. (1968). Fear reduction and fear behavior: Problems in treating a construct. En J. U. H. Shilen (ed.), Research in psychotherapy, vol. 3 (pp. 90-102). Washington: American Psychological Association.

Lang, P. J. (1985). The cognitive psychophysiology of emotion: Fear and anxiety. In A. H. Tuma y J. D. Maser (Eds.), Anxiety and the anxiety disorders (pp. 131-170). Hillsdale, NJ: Erlbaum.

Lawyer, S. R. y Smitherman, T. A. (2004). Trends in anxiety assessment. Journal of Psychopathology and Behavioral Assessment, 26, 101-106.

Lizzereti, N. P., \& Extremera, N. (2011). Emotional Intelligence and Clinical Symptoms in Outpatients with Generalized Anxiety Disorder (GAD). Psychiatry Q, 82, 263-260.

Lizeretti, N. P., Extremera, N., \& Rodríguez, A. (2012). Perceived emotional intelligence and clinical symptoms in mental disorders. Psychiatric Quarterly, 83(4), 407-418. 
Lu, F. J., Li, G. S., Hsu, E. Y., \& Williams, L. (2010). Relationship between athletes' emotional intelligence and precompetitive anxiety. Perceptual and Motor Skills, 110(1), 323-338.

Martínez-Monteagudo, M. C., Inglés, C. J., Granados, L., Aparisi, D., \& García-Fernández, J. M. (2019). Trait emotional intelligence profiles, burnout, anxiety, depression, and stress in secondary education teachers. Personality and Individual Differences, 142, 53-61. doi: https://doi.org/10.1016/j.paid.2019.01.036

Martínez-Monteagudo, M. C., Inglés, C. I., Cano-Vindel, A. y García-Fernández, J. M. (2012). Estado actual de la investigación sobre la teoría tridimensional de la ansiedad de Lang. Ansiedad y Estrés, 18(2-3), 201-219.

Mayer, J. D. y Salovey, P. (1997). What is emotional intelligence? In P. Salovey \& D. Sluyter (Eds.), Emotional development and emotional intelligence: implications for educators (pp. 3-31). New York: Basic Books.

Mayer, J. D., Salovey, P., \& Caruso, D. (2002). Mayer-Salovey-Caruso Emotional Intelligence Test (MSCEIT). Toronto, ON: MHS

Miguel Tobal, J. J., \& Cano Vindel, A. (1997). Manual del Inventario de Situaciones y Respuestas de Ansiedad-ISRA. Madrid: TEA (4ª ed.).

Ministerio de Economía y Competitividad, \& Ministerio de Sanidad, Servicios Sociales e Igualdad (2018). Salud mental y salud pública en España: vigilancia epidemiológica. Madrid: Centro Nacional de Epidemiología.

Peláez-Fernández, M. A., Extremera, N., \& Fernández-Berrocal, P. (2014). Incremental prediction and moderating role of the perceived emotional intelligence over aggressive behavior. The Spanish Journal of Psychology, 17(e15), 1-11.

Pytlik Zillig, L., Hemenover, S., \& Dienstbier, R. (2002). What do we assess when we assess a Big 5 trait? A content analysis of the affective, behavioral and cognitive processes represented in the Big 5 personality inventories. Personality and Social Psychology Bulletin, 28(6), 847-858.

Ruiz, F. J., Suárez-Falcón, J. C., Riano-Hernández, D., \& Gillanders, D. (2017). Psychometric properties of the cognitive fusion questionnaire in colombia. Revista Latinoamericana De Psicología, 49(1), 80-87. doi: 10.1016/j.rlp.2016.09.006

Ruiz-Rodríguez, P., Cano-Vindel, A., Navarro, R. M., Medrano, L., Moriana, J. A., Aguado, C. B., . . . de Investigación PsicAP, G. (2017). Impacto económico y carga de los trastornos mentales comunes en españa: Una revisión sistemática y crítica. Ansiedad y Estres, 23(2-3), 118-123. doi: https://doi.org/10.1016/j.anyes.2017.10.003 
Salguero, J. M., Fernandez-Berrocal, P., Balluerka, N., \& Aritzeta, A. (2010). Measuring perceived emotional intelligence in the adolescent population: Psychometric properties of the trait meta-mood scale. Social Behavior and Personality: An International Journal, 38(9), 1197-1209.

Salovey, P., Mayer, J., Goldman, S., Turvey, C., \& Palfai, T. (1995). Emotional Attention, Clarity, and Repair: Exploring Emotional Intelligence Using the Trait Meta-Mood Scale. En J.W. Pennebaker (Ed), Emotion, Disclosure, \& Health. (pp. 125-154). Washington, American Psychological Association.

Shi, M., Liu, L., Wang, Z. Y., \& Wang, L. (2015). The mediating role of resilience in the relationship between big five personality and anxiety among chinese medical students: A cross-sectional study. PloS One, 10(3), e0119916.

Vazsonyi, A. T., Ksinan, A., Mikuška, J., \& Jiskrova, G. (2015). The big five and adolescent adjustment: An empirical test across six cultures. Personality and Individual Differences, 83, 234-244. doi: http://dx.doi.org/10.1016/j.paid.2015.03.049

Webb, C. A., Schwab, Z. J., Weber, M., DelDonno, S., Kipman, M., Weiner, M. R., \& Killgore, W. D. (2013). Convergent and divergent validity of integrative versus mixed model measures of emotional intelligence. Intelligence, 41(3), 149-156. doi: 10.1016/j.intell.2013.01.004 